\title{
WEAK CONTAINMENT AND WEAK FROBENIUS RECIPROCITY
}

\author{
ELLIOT C. GOOTMAN
}

\begin{abstract}
We study weak containment relations between unitary representations of a group $G$ and a closed normal subgroup $K$ by exploiting a property of $G$-ergodic quasi-invariant measures on the primitive ideal space of $K$. By this means, we prove that every irreducible representation of $G$ is weakly contained in a representation induced from an irreducible representation of $K$ if the quotient group $G / K$ is amenable; and that the pair $(G, K)$ satisfies a weak Frobenius reciprocity property if and only if $G / K$ is amenable and $G$ acts minimally on the primitive ideal space of $K$. If $G / K$ is compact, $G$ acts minimally if and only if the primitive ideal space of $K$ is $T_{1}$.
\end{abstract}

1. Introduction. If $K$ is a closed normal subgroup of a locally compact group $G, G$ acts naturally on PRIM $K$, the primitive ideal space of $K$ endowed with the hull-kernel topology, and, under this action, the system ( $G$, PRIM $K$ ) is a topological transformation group [8, p. 889]. As PRIM $K$ is $T_{0}$, locally compact and second countable (for $K$ second countable), the discussion in [5, pp. 5-7] applies and every $G$-ergodic quasi-invariant probability measure on PRIM $K$ is thus concentrated on a unique quasi-orbit. This observation (also noted in $[12, \S 3])$, applied to the Glimm projection-valued measure [8, Theorem 1.9] associated with the restriction to $K$ of an irreducible representation $\pi$ of $G$, enables us to conclude that the kernel of the restriction of $\pi$ to $K$ equals $\cap_{g \in G} g \cdot J$ for some $J$ in PRIM $K$.

In $\$ 2$ we fill in the details and obtain rather easily the result that if the quotient group $G / K$ is amenable, then every irreducible representation of $G$ is weakly contained in a representation induced from an irreducible representation of $K$. This extends Theorem 4.1-A of [1] and the theorem in [11, p. 314], where the additional hypotheses that $K$ is type I and regularly imbedded in $G$ are required. We conjecture that the above result is true whenever $G$ acts amenably on $G / K$ ( $K$ not necessarily normal) and offer as support a proof in the special case where $G / K$ is compact. Indeed, in this special case weak containment can actually be replaced by strict containment, and an extension of Theorem 4.1-B of [1] is thereby obtained.

In $\$ 3$ we again utilize our basic observation concerning the kernel of the restriction of an irreducible representation of $G$ to a closed normal subgroup $K$ to prove that the pair $(G, K)$ satisfies condition (FP) of Fell [7, p. 442] if and only if $G / K$ is amenable and $G$ acts minimally on PRIM $K$. This latter

Received by the editors May 2, 1974.

AMS (MOS) subject classifications (1970). Primary 22D10, 22D30.

Key words and phrases. Unitary representation, induced representation, weak containment, weak Frobenius reciprocity, primitive ideal space, kernel of a representation, amenable. 
condition means that each orbit closure in PRIM $K$ is a minimal closed $G$ invariant set. The special case of a compact quotient group is considered and a class of examples is given.

In what follows, all groups are assumed to be locally compact, Hausdorff and second countable. By a representation of a group we mean a continuous unitary representation on a separable Hilbert space. $\hat{G}$ denotes the space of equivalence classes of irreducible representations of $G$ and PRIM $G$ the space of primitive ideals of $C^{*}(G)$, the $C^{*}$-algebra of $G$, both equipped with the hullkernel topology. Let $K$ be a closed subgroup of $G, I$ a closed two-sided ideal in $C^{*}(G)$, and $\pi$ and $\sigma$ representations of $G$ and of $K$, respectively. $U^{\sigma}$ denotes the representation of $G$ obtained by inducing $\sigma$ to $G$ [13], $\left.\pi\right|_{K}$ and $\left.\pi\right|_{I}$ denote, respectively, the representation of $K$ obtained by restricting $\pi$ to $K$ and the ${ }^{*}$ algebra representation of $I$ obtained by considering $\pi$ as a representation of $C^{*}(G)$ and restricting. $\mathcal{F}(\pi)$ denotes the Hilbert space of $\pi$, and ker $\pi$ the kernel of $\pi$, considered as a representation of $C^{*}(G)$. The zero space of $\left.\pi\right|_{I}$ is $\{x \in \mathcal{H}(\pi): \pi(i) x=0 \forall i \in I\}$. Recall that for representations $\pi, \rho$ of $G, \pi$ is weakly contained in $\rho$ if and only if $\operatorname{ker} \pi \supseteq \operatorname{ker} \rho[4, \S \S 3.4 .4-3.4 .5]$. We shall write this as $\pi \simeq \underline{\rho}$.

2. Weak containment. Let $K$ be a closed normal subgroup of $G$. $G$ acts by conjugation as a group of automorphisms of $K$, and this action determines in a natural manner an action of $G$ on $C^{*}(K), \hat{K}$ and PRIM $K$ under which $(G, \hat{K})$ and $(G$, PRIM $K)$ are topological transformation groups [8, p. 889].

THEOREM 2.1. For each $\pi$ in $\hat{G}$ there exists $J$ in PRIM $K$ such that $\operatorname{ker}\left(\left.\pi\right|_{K}\right)=\bigcap_{g \in G} g \cdot J$.

Proof. Let $P$ denote the Glimm projection-valued measure associated with $\left.\pi\right|_{K}\left[8\right.$, Theorem 1.9]. Since $P(g \cdot A)=\pi(g) P(A) \pi\left(g^{-1}\right)$ for all $g \in G, A$ Borel $\subseteq$ PRIM $K[2$, Lemma 6], $P$ is quasi-invariant under the action of $G$. As the range of $P$ is contained in the $W^{*}$-algebra generated by $\left.\pi\right|_{K}[8$, Theorem 1.9], it follows from the irreducibility of $\pi$ that $P$ is also ergodic. $\mathcal{H}(\pi)$ is separable (by hypothesis), and thus, there is clearly a separating vector for $P$ and, consequently, a finite positive quasi-invariant ergodic measure on PRIM $K$ with the same null sets as $P$. By [5, pp. 5-7], $P$ is thus concentrated on a unique quasi-orbit $\widetilde{G \cdot J}$ in PRIM $K$, that is, there is some $J$ in PRIM $K$ with $P$ concentrated on

$$
\begin{aligned}
\widetilde{G \cdot J} & =\{I \in \operatorname{PRIM} K: \overline{G \cdot I}=\overline{G \cdot J}\} \\
& =\left\{I \in \operatorname{PRIM} K: \bigcap_{g \in G} g \cdot I=\bigcap_{g \in G} g \cdot J\right\} .
\end{aligned}
$$

Recall that for any $S \subseteq \operatorname{PRIM} K, \bar{S}=\left\{I \in \operatorname{PRIM} K: I \supseteq \bigcap_{J \in S} J\right\}$, and that there is a one-to-one correspondence between closed subsets $C$ of PRIM $K$ and closed two-sided ideals $F$ in $C^{*}(K)$ given by $C=\{I \in \operatorname{PRIM} K: I$ $\supseteq F\}[4, \S \S 3.1 .1-3.1 .2]$. For such a closed set $C, P(C)$ is the projection on the zero space of the representation $\left.\left(\left.\pi\right|_{K}\right)\right|_{F}[2$, p. 1103]. As $P$ is concentrated on $\widehat{G \cdot J} \subseteq \overline{G \cdot J}$, the restriction of $\left.\pi\right|_{K}$ to the ideal $\cap_{g \in G} g \cdot J$ is a zero representation, that is, $\operatorname{ker}\left(\left.\pi\right|_{K}\right) \supseteq \cap_{g \in G} g \cdot J$. If $\cap_{g \in G} g \cdot J \nsupseteq \operatorname{ker}\left(\left.\pi\right|_{K}\right)$, then also 
$J \nsupseteq \operatorname{ker}\left(\left.\pi\right|_{K}\right)$ since $\operatorname{ker}\left(\left.\pi\right|_{K}\right)$ is clearly a $G$-invariant ideal of $C^{*}(K)$. Hence $J \in U \equiv\left\{I \in \operatorname{PRIM} K: I \nsupseteq \operatorname{ker}\left(\left.\pi\right|_{K}\right)\right\}$, a $G$-invariant open set. If $Q$ $\in \widetilde{G \cdot J}$, so that $\overline{G \cdot Q}=\overline{G \cdot J}$, then $J \in \overline{G \cdot Q} \cap U, U \cap G \cdot Q \neq \varnothing$, and by the $G$-invariance of $U, Q \in U$. Thus $\widetilde{G \cdot J} \subseteq U$,

$$
P\left(U^{\prime}=\left\{I \in \operatorname{PRIM} K: I \supseteq \operatorname{ker}\left(\left.\pi\right|_{K}\right)\right\}\right)=0,
$$

and $\left.\pi\right|_{K}$, restricted to the ideal $\operatorname{ker}\left(\left.\pi\right|_{K}\right)$, is nondegenerate. This is clearly absurd, and therefore $\operatorname{ker}\left(\left.\pi\right|_{K}\right)=\bigcap_{g \in G} g \cdot J$.

THEOREM 2.2. Let $K$ be a closed normal subgroup of $G$ such that the quotient group $G / K$ is amenable, and let $\pi \in \hat{G}$. There exists $\sigma \in \hat{K}$ with $\pi \propto U^{\sigma}$.

Proof. By Theorem 2.1 there exists $J$ in PRIM $K$ with

$$
\operatorname{ker}\left(\left.\pi\right|_{K}\right)=\bigcap_{g \in G} g \cdot J .
$$

Choose $\sigma \in \hat{K}$ with ker $\sigma=J .\left.\pi\right|_{K}$ is weakly contained in the $G$-orbit of $\sigma$ and, by [6, Theorem 4.3], $\pi \propto U^{\sigma}$.

We conjecture that whenever $K$ is a closed, not necessarily normal, subgroup of $G$ such that $G$ acts amenably on $G / K$, then the conclusion of Theorem 2.2 holds. We have no results in general, but the following is a strengthening of Theorem 2.2 in a special case.

THEOREM 2.3. Let $K$ be a closed subgroup of $G$ such that $G$ acts amenably on $G / K$ and such that $G / K$ is compact. For each $\pi$ in $\hat{G}$ there exists $\sigma \in \hat{K}$ such that $\pi$ is strictly contained in $U^{\sigma}$.

Proof. For $f$ a continuous function of compact support on $G$, the map $f \rightarrow f \otimes 1$ (considered as a continuous function of compact support on $G \times G / K)$ extends to give an imbedding of $C^{*}(G)$ into the $C^{*}$-algebra $C^{*}((G, G / K))$ associated with the transformation group $G \times G / K \rightarrow G / K$ (see $[9, \S 2]$ for definitions and details and [9, Lemmas 2.2 and 4.1] for a proof). Hence there is an irreducible representation $L=\langle V, M\rangle$ of $C^{*}((G, G / K))$ whose restriction to $C^{*}(G)$, considered as a subalgebra of $C^{*}((G, G / K))$, contains $\pi$ as a subrepresentation [4, Proposition 2.10.2]. As $L(f \otimes 1)=V(f)$ $[9, \S 2], \pi$ is actually a subrepresentation of $V$, and $V=U^{\sigma}$ for some $\sigma \in \hat{K}$ by Mackey's imprimitivity theorem.

3. Weak Frobenius reciprocity. In [7, p. 442], Fell considers the following weak version of the Frobenius reciprocity property (FP): for a closed subgroup $K$ of $G$ and $\pi \in \hat{G}, \sigma \in \hat{K}, \pi \propto U^{\sigma}$ if and only if $\left.\sigma \propto \pi\right|_{K}$. For a closed normal subgroup $K$ of $G$, we apply Theorem 2.1 to derive necessary and sufficient conditions on the pair $(G, K)$ in order that it satisfy (FP). We consider separately the following conditions:

$$
\left.\sigma \propto \pi\right|_{K} \Rightarrow \pi \simeq U^{\sigma}, \quad \forall \pi \in \hat{G}, \quad \sigma \in \hat{K},
$$

and

$$
\left.\pi \propto U^{\sigma} \Rightarrow \sigma \underline{\propto} \pi\right|_{K}, \quad \forall \pi \in \hat{G}, \quad \sigma \in \hat{K} .
$$

Definition. An ideal $J$ in PRIM $K$ is said to be $G$-primitive if there exists $\pi \in \hat{G}$ with $\operatorname{ker}\left(\left.\pi\right|_{K}\right)=\bigcap_{g \in G} g \cdot J$. 
Lemma 3.1. Let $K$ be a closed normal subgroup of $G$. The pair $(G, K)$ satisfies (FP1) if and only if $G / K$ is amenable and the $G$-orbit closures of $G$-primitive ideals in PRIM $K$ are minimal, that is, minimal closed $G$-invariant sets.

Proof. $(\Rightarrow)$ Let $(G, K)$ satisfy (FPl). Letting $\pi$ and $\sigma$ be the identity representations of $G$ and $K$, respectively, we have that $G / K$ is amenable. For a $G$-primitive ideal $J$ in PRIM $K$ and $\pi \in \hat{G}$ such that $\operatorname{ker}\left(\left.\pi\right|_{K}\right)=\bigcap_{g \in G} g \cdot J$, choose any $P \in \overline{G \cdot J}$ and $\rho \in \hat{K}$ with $\operatorname{ker} \rho=P .\left.\rho \subseteq \pi\right|_{K}$, hence, $\pi \simeq U^{\rho}$ and

$$
\bigcap_{g \in G} g \cdot J=\operatorname{ker}\left(\left.\pi\right|_{K}\right) \supseteq \operatorname{ker}\left(\left.U^{\rho}\right|_{K}\right)=\bigcap_{g \in G} g \cdot P
$$

[6, Lemma 1.11 and the proof of Theorem 4.3]. Thus for $P \in \overline{G \cdot J}$ we have $\overline{G \cdot J} \subseteq \overline{G \cdot P}$, and the orbit closure of $J$ is clearly minimal.

$(\Leftarrow)$ Let $\pi \in \hat{G}, \sigma \in \hat{K}$ with $\left.\sigma \propto \pi\right|_{K}$. By Theorem 2.1,

$$
\operatorname{ker}\left(\left.\pi\right|_{K}\right)=\bigcap_{g \in G} g \cdot J
$$

for some (a priori) $G$-primitive $J \in \operatorname{PRIM} K$. ker $\sigma$ is a primitive ideal in the orbit closure of $J$ and thus, by hypothesis, $\bigcap_{g \in G} g \cdot J=\cap_{g \in G} g \cdot \operatorname{ker} \sigma$. As $g \cdot \operatorname{ker} \sigma=\operatorname{ker} g \cdot \sigma,\left.\pi\right|_{K}$ is thus weakly contained in the $G$-orbit of $\sigma$, and by [6, Theorem 4.3] (recall that $G / K$ is amenable), $\pi \propto U^{\sigma}$.

We have no necessary and sufficient conditions on $(G, K)$ ensuring that (FP2) is satisfied. We do have, however,

LEMMA 3.2. Let $K$ be a closed normal subgroup of $G$ such that $G / K$ is amenable. Then the pair $(G, K)$ satisfies $(\mathrm{FP} 2)$ if and only if $G$ acts minimally on PRIM $K$.

Proof. $(\Rightarrow)$ We first show that every ideal in PRIM $K$ is $G$-primitive. Choose $J \in \operatorname{PRIM} K, \sigma \in \hat{K}$ with $\operatorname{ker} \sigma=J$ and $\pi \in \hat{G}$ with $\pi \propto U^{\sigma}$. By Theorem 2.1, ker $\left.\pi\right|_{K}=\cap_{g \in G} g \cdot Q$ for some $Q$ in PRIM $K$, and thus $\bigcap_{g \in G} g \cdot J \supseteq \cap_{g \in G} g \cdot Q$ by hypothesis. By Theorem 4.3 of [6], ker $\left.\pi\right|_{K}$ $\supseteq \bigcap_{g \in G} g \cdot J$. Hence $\overline{G \cdot J}=\overline{G \cdot Q}$ and $J$ is $G$-primitive since $Q$ is. Now choose $J, Q \in \operatorname{PRIM} K$ and $\pi \in \hat{G}, \sigma \in \hat{K}$ so that $Q \in \overline{G \cdot J}$, $\left.\operatorname{ker} \pi\right|_{K}$ $=\bigcap_{g \in G} g \cdot Q$ and ker $\sigma=J . \pi \propto U^{\sigma}\left[6\right.$, Theorem 4.3], hence, $\left.\sigma \propto x\right|_{K}$ by hypothesis and $\overline{G \cdot Q}=\overline{G \cdot J}$.

$(\Leftarrow)$ Let $\pi \in \hat{G}, \sigma \in \hat{K}$ with $\pi \propto U^{\sigma}$. If ker $\sigma=J$ and

$$
\operatorname{ker}\left(\left.\pi\right|_{K}\right)=\bigcap_{g \in G} g \cdot Q
$$

for $Q \in \operatorname{PRIM} K$ (Theorem 2.1), then $\cap_{g \in G} g \cdot Q \supseteq \cap_{g \in G} g \cdot J \quad[6$, Lemma 1.11 and the proof of Theorem 4.3], $J \supseteq \cap_{g \in G} g \cdot Q$ by minimality, and $\left.\sigma \underline{\simeq} \pi\right|_{K}$.

THEOREM 3.3. Let $K$ be a closed normal subgroup of $G$. Then the pair $(G, K)$ satisfies (FP) if and only if $G / K$ is amenable and $G$ acts minimally on PRIM $K$.

Proof. Combine Lemmas 3.1 and 3.2.

THEOREM 3.4. If $K$ is a closed normal subgroup of $G$ with $G / K$ compact, the pair $(G, K)$ satisfies (FP) if and only if PRIM $K$ is $T_{1}$. 
Proof. As compact groups are amenable, we need only show that $G$ acts minimally on PRIM $K$ if and only if PRIM $K$ is $T_{1}$. Accordingly, assume PRIM $K$ is $T_{1}$ and let $Q, J \in \operatorname{PRIM} K$ with $Q \in \overline{G \cdot J}$. Hence, $Q=\lim g_{\gamma} \cdot J$ in PRIM $K$, and since $K$ acts trivially on PRIM $K$ and $G / K$ is compact, we may assume without loss of generality that $g_{\gamma} \rightarrow g$ in $G$. By [8, p. 889], $J=g_{\gamma}^{-1} \cdot\left(g_{\gamma} \cdot J\right) \rightarrow g^{-1} \cdot Q$ in PRIM $K$, that is, $g^{-1} \cdot Q \in \bar{J}$. If PRIM $K$ is $T_{1}$, this implies $Q=g \cdot J$, and, in fact, orbit closures are actually orbits and are clearly minimal. Conversely, suppose $G$ acts minimally on PRIM $K$ and let $Q, J \in$ PRIM $K$ with $Q \supseteq J$. Let $P$ be a minimal primitive ideal contained in $J$ [3, Corollary 2 , p. 100]. We shall prove $P=Q \cdot Q \in \bar{P} \subseteq \overline{G \cdot P}$ and by minimality of orbit closures, $P \in \overline{G \cdot Q}$. Using the compactness of $G / K$ as above, we have $P \supseteq g \cdot Q$ for some $g \in G$. Now $Q \supseteq P \supseteq g \cdot Q \supseteq g \cdot P$, $P=g \cdot Q=g \cdot P$ by minimality of $P$ and, hence, $P=Q$.

Corollary 1. Let $K$ be a closed normal type I subgroup of $G$ with $G / K$ compact. The pair $(G, K)$ satisfies $(\mathrm{FP})$ if and only if $K$ is $C C R$.

Proof. As is well known, if $K$ is type I, then $\hat{K}$ and PRIM $K$ are homeomorphic, and $\hat{K}$ is $T_{1}$ if and only if $K$ is CCR.

REMARK. Corollary 1 justifies the feeling that (FP) is probably satisfied only in the presence of conditions either akin to, or generalizing, compactness. In the presence of the strongest possible such condition, namely that $G$ be compact, we have of course the best possible result, the classical Frobenius reciprocity theorem.

COROllary 2. Let $G$ be a connected simply connected Lie group and $S$ its solvradical. Then $(G, S)$ satisfies (FP) if and only if $G$ is amenable and all the roots of the Lie algebra of $S$ are purely imaginary.

Proof. A solvable group $S$ is always amenable. Hence, $G$ is amenable if and only if $G / S$ is, and the semisimple group $G / S$ is amenable if and only if it is compact [10, pp. 30 and 53]. The result follows by Theorems 3.3 and 3.4, and Theorem 2 in $\S 8$ of [14].

\section{REFERENCES}

1. L. Baggett, $A$ weak containment theorem for groups with a quotient $R$-group, Trans. Amer. Math. Soc. 128 (1967), 277-290. MR 36 \#3921.

2. R. J. Blattner, Group extension representations and the structure space, Pacific J. Math. 15 (1965), 1101-1113. MR 32 \#5785.

3. J. Dixmier, Sur les $C^{*}$-algèbres, Bull. Soc. Math. France 88 (1960), 95-112. MR 22 \# 12408.

4. - Les $C^{*}$-algèbres et leurs représentations, Cahiers Scientifiques, fasc. 29, GauthierVillars, Paris, 1964. MR 30 \# 1404.

5. E. G. Effros and F. Hahn, Locally compact transformation groups and $C^{*}$-algebras, Mem. Amer. Math. Soc. No. 75 (1967). MR 37 \#2895.

6. J. M. G. Fell, Weak containment and induced representations of groups, Canad. J. Math. 14 (1962), 237-268. MR 27 \#242.

7. - Weak containment and induced representations of groups. II, Trans. Amer. Math. Soc. 110 (1964), 424 447. MR 28 \#114.

8. J. G. Glimm, Families of induced representations, Pacific J. Math. 12 (1962), 885-911. MR $26 \# 3819$. 
9. E. C. Gootman and R. R. Kallman, The left regular representation of a p-adic algebraic group is type I, Advances in Math. (to appear).

10. F. P. Greenleaf, Invariant means on topological groups and their applications, Van Nostrand Math. Studies, no. 16, Van Nostrand, Princeton, N. J., 1969. MR 40 \#4776.

11. Amenable actions of locally compact groups, J. Functional Analysis 4 (1969), 295\#315. MR $40 \# 268$.

12. R. Howe, On representations of discrete, finitely generated, torsion-free, nilpotent groups (preprint).

13. G. W. Mackey, Induced representations of locally compact groups. I, Ann. of Math. (2) 55 (1952), 101-139. MR 13, 434.

14. L. Pukanszky, The primitive ideal space of solvable Lie groups, Invent. Math. 22 (1973), 75118.

Department of Mathematics, University of Georgia, Athens, Georgia 30602 